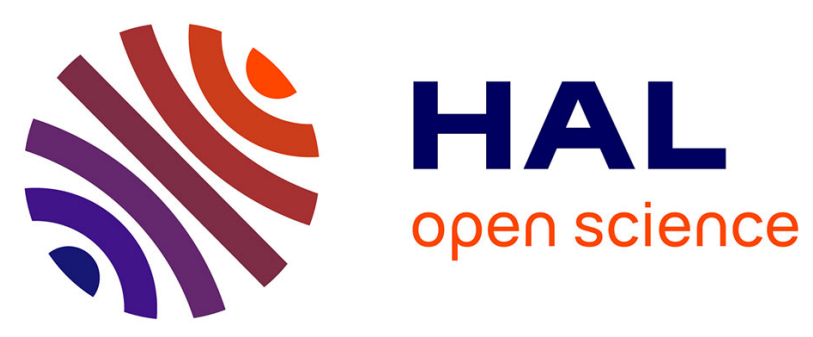

\title{
Quand les agriculteurs et les étudiants pensent l'objet pesticide : analyse discursive des représentations sociales
}

\author{
B. Zouhri, Karine Weiss, E. Garros-Levasseur, A. Valette
}

\section{To cite this version:}

B. Zouhri, Karine Weiss, E. Garros-Levasseur, A. Valette. Quand les agriculteurs et les étudiants pensent l'objet pesticide : analyse discursive des représentations sociales. Pratiques Psychologiques, 2016, 22 (3), pp.221 - 237. 10.1016/j.prps.2016.05.002 . hal-01644873

\section{HAL Id: hal-01644873 \\ https://hal-amu.archives-ouvertes.fr/hal-01644873}

Submitted on 9 May 2018

HAL is a multi-disciplinary open access archive for the deposit and dissemination of scientific research documents, whether they are published or not. The documents may come from teaching and research institutions in France or abroad, or from public or private research centers.
L'archive ouverte pluridisciplinaire HAL, est destinée au dépôt et à la diffusion de documents scientifiques de niveau recherche, publiés ou non, émanant des établissements d'enseignement et de recherche français ou étrangers, des laboratoires publics ou privés.

\section{(ㅇ)(1) $\$$}

Distributed under a Creative Commons Attribution - NonCommercial - NoDerivatives| 4.0 
Psychologie sociale

\title{
Quand les agriculteurs et les étudiants pensent l'objet pesticide : analyse discursive des représentations sociales
}

When famers and students think about pesticides: Discoursive analysis

\author{
B. Zouhri ${ }^{a, *}$, E. Garros-Levasseur ${ }^{b}$, K. Weiss ${ }^{c}$, A. Vatette
}

a Labontmive de pspchologic sociale d'Aix-Marseille-unirersite (EA 849), 29, avenue Robert-Sciuman. $1362 /$ Aix-en-Provence, France

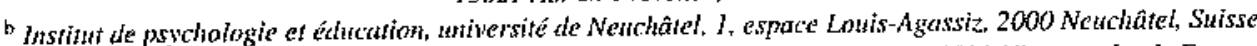

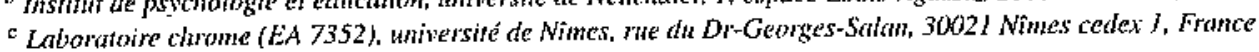

\section{Résumé}

Celte étude s'intéresse à la manière dont les agriculteurs et les étudiants en lycée angricole représentent les pesticides. Comment ils perçoivent cet objet, comment ils le pensent et comment ils l'utilisent en fonetion de Ieur tieu d'habitation (Paca/Languedoc-Roussillon, Bretagne en Martinique) ef leur type d'implication (étudiants vs agriculteurs). Pour ce faire, une enquête qualitative (entreticns semi-dircetifs) a ćté menće anprès de 77 personnes vivant dans !'une des trois régions: et analysce à l'aide d'un logiciel d'alaalyse de donnécs textuelles (Iramuleq). Les résultats soulignent une représentation sociale des pesticides qui s'organise autour de ja notion de risque sanitaire concenant les agrjculteurs ainsi que les risques environtementaux lićx à l'utilisation des pesticjues.

๑) 2016 Société Française de Psychologie. Publie par Elsevier Masson SAS. Tous droits réservés.

Mots cles: Pesticide ; Representation socialt: ; Anajyse textuetle; Risques

\section{Abstract}

The present sudy was interested in how professional farmers and students of an agricultural secondary school represent pestictides. More precisely, how in Junction of their place of residence in France (Paca/Languedoc-Roussilon, Bretagne and Martinique) and their implication level with the farming activity

\footnotetext{
* Auleur colrespondanl.

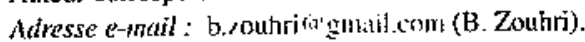


(students vs. professional farmers) fo they perceive and think about pesticides. To do so, 77 participants living in one of the above-mentioned regions responded to a qualitative inquiry, which was anatysed through Iramuteq - a software used for multidimensional lext and questionnaire analysis. Results suggest that the pesticide social representation is mainly organised around $(1)$ the tdea that pesticides present sanilary riskss for farmers; and (2) the idea that pesticides irwolve environmental risks.

O 2016 Société Française de Psychologie. Published by Elsevier Masson SAS. All rights reserved.

Kcyword: Pusticide; Sociå representation: Texual anatysis; Risks

\section{L'agriculture aujourd'hui : un contexte propice à l'étude des représentations sociales}

La révoluzion verte, initiée dans les années 1960 , correspondait à unc politique intensive de đéveloppement de l'agriculture permeltant d'augmenter par trois les rendements, et ce sans augmentation significative des surfaces agricoles. Cette révolution permit d'augmenter les rendements et la productivité de façon significative entre les années 1960 et 1990, avec unẹ artịculation autour de trois grands axes:

- sélection de variétếs à haut rendement;

- meilleure maîtrise de l'eau ;

- généralisation de l'utilisation des produits plyytosanitaires.

Ce succès économique laisse, 50 ans après, un goût amer sar l'ensemble du monde agricole. L'ensemble des dispositions prises à cette époque a favorisé la disparition des petites cultures, réduit la biodiversité et abouti à une dégradation des terres via đ'utilisation effrénée des intrants. L'héritage de cette révolution verte a fait émerger des demandes sociétales pour une agriculture plus respectueuse de la santé et de l'environnement. Des décisions politiques doivent alors être prises pour faire face à ces nouvelles préoccupations environnementales et sanitaires. Lancé initialement à la suite du Grenelle de l'environnement en 2008, le plan Ecophyto a pour principa] objectif une diminution de $50 \%$ de l'utilisation des produits plyytosanilaires à l'horizon 2018 , aussi bien dans les zones agricoles gu'en milieu urbain. Néanmoins, avec une augmentation de $5 \%$ entre les années 2009 et 2013 , et de $9 \%$ entre 2012 et 2013 , l'utilisation des produits phytosanitaires dans le domaine de l'agriculture n'a de cesse d'augmenter depuis ces six dernières années. L'ambition affichée du plan n'est visiblement pas si simple: « six ans après sou démarrage fin 2008 , le plan n'a pas eu les résultats espérés puisque les indicateurs de suivi ne montrent pas de tendance à la baisse » (Potier, 2014, p. 1 i). C'est dans ce contexte que le ministre de l'Agriculture, en janvier 2015, annonce une nouvelle version du plan, Ecophyto V2, visant une réduction de $50 \%$ des pesticides à l'horizon 2025 . Le défi de l'ensemble de ces politiques publiques réside dans cette double volonté: maintenir au même niveas une production agricole significative et répondre, en même temps, aux exigences sanitaires et environnementales (Aubertot et a]., 2005: Busca, 2016). Ceci appelle à un changement de pratiques et pose la question de l'information, de la sensibilisation et des incitations à mettre en cuvre pour faciliter ce clüngement. Toutefois, la réduction de l'utilisation de produits phytosanitaires ne peut être atteinte sans prendre en compte le point de vue des utilisateurs. 
En effet, pour qu'un groupe s'engage dans une pratique plutôt qu' une autre, il faut que celte dernière soit en coluérence avec les valeurs et les normes de son groupe: «Il ne suffit pas qu'un individu soit engagé dans une pratique pour qu'il la reconnaisse eomme sienne el se l'approprie. Encore faut-il qu'elle lui apparaisse comme acceptable par rapport au système de valeurs qui est le sien " (Abric, 1994, p. 220). C'est dans l'interaction entre culture et processus cognitifs que se jouent les pratiques collectives, terreaux d'expression identitaire des groupes (Péres. 2004), En outre, de nombreux travaux mettent en exergue l'importance du niveau de connaissance de l'objet comme variable explicative des pratiques et de la construction des représentations sociales (Moscovici, 1961: Moliner. 1998).

Dans cet objectif́, il semble pertinent de saisir des informations et connaissances émanant des agriculteurs eux-mêmes, afun de construire par la suite des programmes de sensibilisation s'appuyant sur ces éléments : quels discours tiennent-ils à propos des pesticides, de leur ufilisation, de leur dangerosité ? Conment envisagent-ils la diminution de l'emploi de produits phytosanitaites? Quelles pratigues ajternatives sont connues, imaginées, attendues, etc. ?

Les pesticides, en tant qu'un objet social, ctistallisent des investissements différents en fonction des groupes et permettent par la même l'émergence de processus sociocognitifs spécifiques. Notons égalennent, l'existence de pratiques plurietles concernant leurs usages s'inscrivant datns des cadres socioculturels spécifịques. Cel ensenble de caractéristiqures contribue à asseoir la pertinence de l'étude des pesticides sous le prisme de la théorie des représentations sociales en tant gुu'objet social polémique.

En effer, s'intéresser aux représentations sociales, c'est porter notre intérêt sur « une forme de connaissance spécifique, le savoir de sens commun, dont les contenus manifestent l'opération do processits génératís et fonctionneis socialement marqués » (Jodelet. 1984, p. 361). Les représentutions sociales sont des modalités de connaissances, collectivement produites, « contribuant aux processus d'orientations des conduites» (Moscovici. 1961). C'est bien cette fonction de mode đ'emploi de la réalité sociale (Mvliner, 1995) quá nous intéresse plus particulièrement dans le cadre de cette étude.

\section{De la pertinence du recours à la théorie des représentations sociales}

De nombreux auteurs s'accordent pour mettre en évidence les différentes conditions relatives a l'émergence d'objet de représentation sociale. Notons les travaux de Moliner ( (993) qui retient Irois conditions pour qu'un objot se constitue en objet de representation sociale. Pour qu'un objet lajsse prise à un processus d'élaboration socioreprésentationnel, il doit d'abord être polymorphe, c'est-à-dite complexe par sa nature, mais aussi complexifié par le traitement que la pensée sociale lıi inflige. De plus, cet objet ne prend son sens que dans sa relation à un groupe social défini. Les cognitions relatives à l'objet doivent être partagées par les membres d'un même groupe, et par là-même valider «qu'une représentation sociale est un ensenble organisé de cognitions relatives à un objet, partagé par les memb̀res d'une population homogène par rapport à cet objet ” (Flament, 1994, p. 60). L'objet doit ensuite être structurant pour le groupe : il peut permettre soit de structurer l'identité du groupe (Zothri \& Rateau, 2015), soit d'çre appréfendé par le groupe daus un nouveau contexte. De plus, des enjeux communs doivent également être mis en évidence, ces derniers « déterminant des objectifs collectifs entendus conme somme d'objectifs individuels " (Moliner, 1496, p. 30).

Notons également les travaux de Hilument et Rouquette (2003), qui s'attachent à deux prérequis relatifs à l'apparition d'une représentation sociale, rappelant ainsi que son «lien avec la société est fondamental " (p. 32). Le premicr principe, qui est à la croisée de deux propriétés complexes, 
est la saillance sociocognitive. D'une part, l'objet doit être un concept générique et suffisatmment abstrait, c'est-à-dire qu'il doit renvoyer à une classe génćrale; ici, l'objet pesticide renvoie aux fongicides, herbicides, insecticide et parfois mêtne OGM. D'autre part, l'objet doit être récurrent dans la communication interpersotnelle et intergroupe. Sa circulation dans la communication et son exposition médiatique, impliquant alors prises de positions et installation de polémique, sont importantes. L'objet doit ctre complètement plongé dans les processus de communication et être imprégné d'avis, d'opinions, de jugements. Autrement dit, pour qu'il y ait représentation, il faut que l'objet pénètre la vie des individus et que les individus fassent pénétrer l'objet darns leur sphère sociale par la pratique qui lui est associéc.

L'ensemble de ces caractéristiques nous invite à penser les pesticides conme objet sociai, sensible et propice à une analyse psychosociale au travers dc la théorie des représentations sociates. Ull de ses fondements est de considérer que le sens commun répond à sa propre logique, une logique naturelle, et que, en ce sens, il n'est pas irrationnel (Moscovici, 1484).

Dès lors, l'objectif principal de cette ćtude est de mettre en évidence les représentations sociales des pesticides à travers l'analyse des discours de différents groupes d'agriculteurs et étudiants en lycée agricole. Plusieurs facteurs sont pris en compte dans cette analyse. Ces groupes se distinguent par leur expérience professionnelle en lien avec leur type d'implication (étudiants vs agriculteurs) et le mode de production développé, à savoir de l'agriculture conventionnelle, raisonnée ou biologique, mais aussi par leur lieu d'habitation (Paci/Languedoc-Roussillon, B retagne et Martinique) qui peut être associé aussi à des discours et des pratiques spécifiques vis-à-vis des pesticides de par leur histoire ou la prédominance d’un certain lype de culture.

\section{Méthodologie}

Une méthode qualitative teile que l'entretien est adaptée à l'objectif principal de l'étude dans le sens où elfe permet de recueillir ce que pensent des individus à propos d'un sujet (Iilick. Fuster, \& Cuillaud, 2015). L'objectif principal des entretiens est de recueillir les théories naïves des sujets et d'analyser la façon dont ils les formulent, de mettre en exergue les discours relataifs à l'utitisation des pesticides, pratiques réelles mais aussi pensées. Ainsi, lors de ce travail de recherche, nous avons pris le parti de nous intéresser à detux groupes refétant différents niveaux de pratique concernant les pesticides : des agriculteurs en activité el des étudiants en lycée agricole. En effet, les sujets ayant une pratique de l'objet ont une représentation plus structurée (Rateau d' Rouquctte, 1998) et mobilisent donc les fonctions descriptives et fonctionnelles (Abric \& Tufini, 1945). Conjointement, avec une moindre pratigue, la représentation sociale est moins structurée é mobilise done les dimensions évaluatives de l'objet (Abric \& fafani, 1995). Une grille d'entrelien a été préalablement construite. Son élaboration s'appuie, d'une part, sur des travaux concernant les représentarions sociales du risque (Micbel-Guillot, 2011 ; Michet-Guillou \& Weiss, 2007), et d'autre part, sur l'ensenble des thématiques abordées dans la littérature au sujet des agriculteurs et de leurs utilisations des pesticides.

La grille d'entretien a ainsi été structurée autour dess principales thérnatiques suivastes:

- pratique/métier (ou formation pour les étuciants): description du métier ou de ta formation; connaissance/engagement dans des groupements agricoles et/ou dans des démarches pro-environnementales;

- utilisation des pesticides: utilisation des pesticides, déterminants du choix des pesticides, conseils daus $1^{*}$ utilisation des produits ; 
Tableau 1

Répartition de l"échantillon selon les variables a territoire agricoles, agenre s al a lype de producten $\%$.

\begin{tabular}{|c|c|c|c|c|}
\hline & Brolagne & Martinique & Sud & Totaux \\
\hline \multicolumn{5}{|c|}{ Agriculowe comentionnelle } \\
\hline Agriculitice & 0 & 7 & 0 & 7 \\
\hline Agricullegr & 4 & 3 & 7 & 14 \\
\hline Sous-tolal AC & 4 & 10 & 7 & 21 \\
\hline \multicolumn{5}{|c|}{ Agricultrent trisonné } \\
\hline Agricultrice & 0 & 0 & 1 & I \\
\hline Agriculteur & 3 & 0 & 0 & 3 \\
\hline Sous-1otal AR & 3 & 0 & l & 4 \\
\hline \multicolumn{5}{|c|}{ Agriculare biologtode } \\
\hline Agricultrice & 1 & 0 & 3 & 4 \\
\hline Agriculteur & 4 & 4 & 1 & 9 \\
\hline Sous-lotal BIO & 5 & 4 & 4 & 13 \\
\hline Etridiante & 7 & 4 & I & $\mathrm{L} 2$ \\
\hline Etudian & 8 & 11 & 8 & 27 \\
\hline Sous-lotal ET[] & {$[5$} & 15 & 9 & 39 \\
\hline
\end{tabular}

- risques liés à l'utilisation des pesticides : types de risques identifiés, description des risques, populations touchées, connaissance de la réglementation, information.

Soixante-dix-sept personnes ont êté interviewées selon cette grille d'entretien. Les interviewés sont des agriculteurs et des étudiants en lycées agricoles dans trois zones aux caractéristiques géoclimatiques différentes et sélectionnées pour leurs spécificités en termes d'utilisations des pesticides:

- sud de la France (régions PACA et Languedoc-Roussillon);

- Bretagne:

- Martinique.

Le Tablèa I présente la répartition de l'échantillon des interviewés.

L'analyse lexicométrique đes entretiens: les entretiens s'articulent autour des qutestions de l'interviewer posées systématiqutement et qui suivent de près l'ordre chronologique de la grille d'entretien. L'ensemble des discours a été retranserit, formaté afin d'effectuer une analyse lexicométrique basée sur la méthode définie par Reineri (1483. 1999, 2003) et réalisée à l'aide du logiciel Iramutę (Ratinaud. 2009). Nous présentons la codification utilisée dans la suite de cette partie.

Cette méthode, basée sur une classificatson hiérarchiqute descendante, est une des méthodes privilégiées permettant l'uccès à l'organisation et au contenu d'une représentation (Abric, 2003). Elle a été éprouvée dans diférentes études portant sur différentes représentations sociales (MichelGuillou, Lalanne \& Krien, 2015; Klein \& Licata. 2003; Lahlou, 2001; Kalampalikis, 2003 : Masson. 2002). Cette méthode permet de mettre en évidence les traces lexicales les plus importantes dans le corpus, autrement dit de révéler les mondes texicaux le structurant. L'hypothèse sous-jacente est que la répétition des traces lexicales est liée à l'intention de faire sens. Il s'agit d'une analyse pragmatique du discours (Kilampalikis \& Moscovici, 2005). Ainsi, lors de l'analyse, une distribution/classification des enoncés s'opère selon la redondance des traces lexicales, mesurée par un $\mathrm{Chi}^{2}{ }^{2}$. L'organisation topique du discours est alors déterminée à travers 
Tableat 2

Coulage des variables thématiques issues de la grilfe d'entretien.

\begin{tabular}{|c|c|}
\hline Thémaliqutu: & Codige thematique \\
\hline Descriplion dil metier & * Múlter \\
\hline Deseription de la formation & -FForm \\
\hline Fłulut tintítier & _*Fulur_mulies \\
\hline Conmissance des groupements agricoles & *Conn_group_agi \\
\hline Connaissunce des déplarclaes pro-environnementalt: & _*Dem_pro_enviro \\
\hline Utilisation des pesticides & -*Util_pes! \\
\hline Assocjations de mots a pesticjules & *Assoc_nots \\
\hline Averie des pesticides & $-^{*}$ Avenir_pest \\
\hline Rirques liés à l'usage des pesticides & - I=Risq_util_pest \\
\hline Regglenentalion liéc à l'usage des pesticides & -HReglem_pesl \\
\hline Conseils sur le choix des pesticides & -FConseil_pust \\
\hline
\end{tabular}

fa mise en évidence des mondes lexicaux qu'elle cartographie (Reinert. 1999). Une fots l'analyse séalisée par le logiciel, le travail d'interprétation reste entier. Il faut donner un sens aux mondes lexicaux el ainsi procéder à l'interprétation des résuliats. Pour cela, nous nous référons pour chaque classe aux formes significativement présentes et absentes indiguées selon leur Chi ${ }^{2}$.

Cette méthode permet egalement de confronter la répartition des énoncés ou segments de textes (UC) selon des variables préalablement assignées aux textes (ou UCI). Ces variables sont externes, c'est-ã-dire fixées par l'utilisateur diu logiciel. Ce codage supplếmentaire permet de tester ult certain nombre d'hypothèses par rapport au corpus. Ainsi, plusieurs variables et modalités ont été défuries avant l'analyse du corpus d'entretien. Tout d'abord, les entreticns ont été codés selon les critères retenus pour discriminer la population interrogée, à savoir le genre, la population, le territoire agricole et le type de productions.

Pour parfaire le codage, les variables tlématiques correspondant aux différents thèmes de la grille d'entretien ont également été définies. Ce codage permet de réduire le poids des mots de f'interviewer dans lit constitution des classes, sans pour autant occulter l'influence de la grille d'entretien. De plus, ce codage permet de repérer pour quelse âématique un type d'énoncé est plus significativement formulé. Le 'a 'ahleáu 2 présente le codage des questions pour les deux grilles d'entretien.

Une fois le codage du corpus effectué, l' analyse lexicométrique a pu ctre menée avec le logiciel Iramuteq. Cette analyse correspond an une classification simple sur segments de tex te standard avec lemmatisation.

\section{Résultats}

Au-delà de la description de classes, un des intérêts de l'analyse issue d'une classification hiérarchique descendante (méthode Reinert) est de dévoiler les liạisons, les rapports entre les mondes lexicaux. Afin de comprendre les regroupements entre les mondes lexicaux el de procéder à teur interprétation, on s'appuie sur les formes significativement présentes garss les classes calculées selon les $\mathrm{Cli}^{2}$.

La Fig. I ofíre une representation graphique de la distribution des mondes lexicaux.

On note tout d'abord que la classe 6 se différencie très tôt des autres classes. Elle représente $20,4 \%$ des segments classés. Les formes les plus significatives de cette classe donnent 


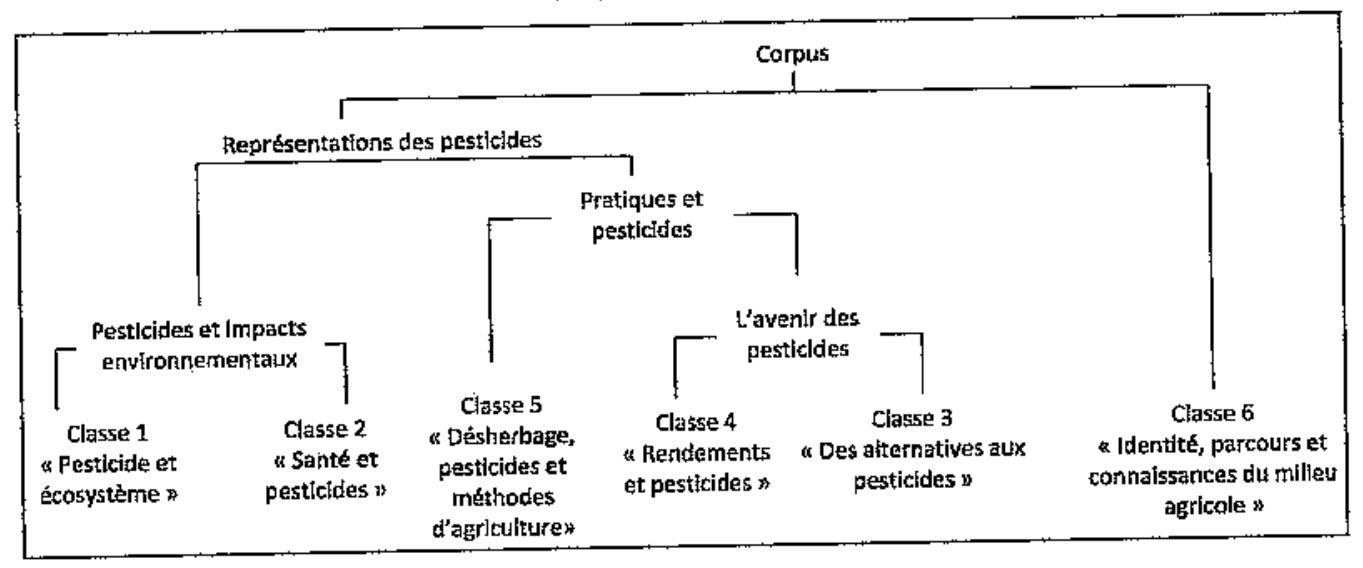

Fig. 1. Dendrogramme avec classes et axes notmmés.

des informations sur son contenu. Les formes formation $\left(\mathrm{Chi}^{2}=294,3, p<0,001^{1}\right)$, groupement $\left(\mathrm{Cli}^{2}=88,46\right)$, BTS $\left(\mathrm{Chi}^{2}=218,15\right)$, certi-phyto $\left(\mathrm{Chi}^{2}=122,04\right)$, métier $\left(\mathrm{Chi}^{2}=83,59\right)$ sont liées aux questions de ta première partie des grilles d'entretien intitulée «Pratique/métier ou Formation \%. L' 'analyse de cette classe, du point de vie de l'organisation spatiale du discours, ne permet pas d'obtenir de plus amples informations. Toutefois, on peut souligner que ces éléments liés à l’identité de l'interviewê, à sa formation, sont présents de manière significative uniquement dants celte classe. Or, on aurait pu par exemple s'attendre à ce que le discours sur la connaissance des groupements agricoles ou sur les démarches pro-environnementales soient associées au discours sur les pratiques vis-à-vis des pesticides (présent dans les classes $3,4 \mathrm{et} 5$ ). En effet, les résulats indiquent que les agriculteurs tout comme les étudiants s'appuient très peu sar les connaissances acquises au cours de leur formationt ou à travers certains groupes agricoles dans l'élaboration de leurs discours à propos des pesticides.

Les 5 autres classes se distinguent ensuite en deux groupes. D'une part, les classes 1 et 2 qui rassemblent des mondes lexicaux associés aux conséquences de l'utilisation des pesticides, et d'autre part, les classes 3,4 et 5 qui concernent davantage les pratiques des agriculteurs. Le contenu de ces classes est présenté dans le Tableạ 3 et détaillé ci-après.

\subsection{Pesticides et impacts environnementaux}

Lintégralité des entretiens traite de la représentation des pesticides (avec un effectif total de 697 , c'est le mot le plus présent dans le corpus) en abordant différentes sous-thématiques. Les classes 1 et 2 sont focalisées sur le rapport entre pesticides et environnement, incluant les hommes, la faune et la flore. Les formes actives * environnement " $\mathrm{el}$ " pesicides " figurent parmi les Chi ${ }^{2}$ les plus élevés dans ces deux classes, à l'opposé des autres classes.

\section{I.I. Les pesticides néfastes: the réaction en chaûne-terre vivante/terre caltivéé}

La classe $\mathrm{L}$ «pesticides et écosystèmes » est principalement liée aux territoires agricoles de Martinique $\left(\mathrm{Chi}^{2}=35,25\right)$ et de Bretagne $\left(\mathrm{Chi}^{2}=23,23\right)$. Elle est trìs largernent liée à la variable

\footnotetext{
${ }^{1}$ Les Chi ${ }^{2}$ indiqueses dans cel anticte sont tous significatils à $p<0,00 \mathrm{t}$.
} 
Tableau 3

Formes lexicales significnlives ol Clit $^{2}$ des classes thématiques 1 à 5

\begin{tabular}{|c|c|}
\hline $\overrightarrow{C l a s s e}$ ( $\mathrm{s}^{\circ}$ ef thémiatique) & Founes lexicales signiticatives $\left(\mathrm{Chi}^{2}\right)$ \\
\hline t. Peslicides el ecosyslème & 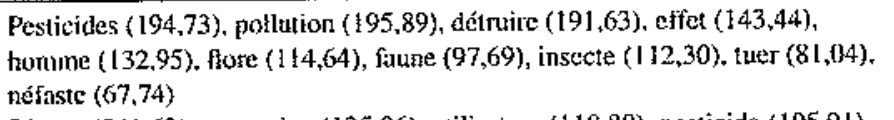 \\
\hline 2. Santé el pestictdes & $\begin{array}{l}\text { Risque }(24), 63) \text {, protection }(135,06) \text {, uilisateur }(118,80) \text {, pesticide }(105,91) \text {, } \\
\text { consommateur }(101,18) \text {, hemain }(81,70) \text {, eatu }(81,03) \text {, réglementation }(80,49) \text {, } \\
\text { dose }(53,99)\end{array}$ \\
\hline 3. Allematives aux pesticides & 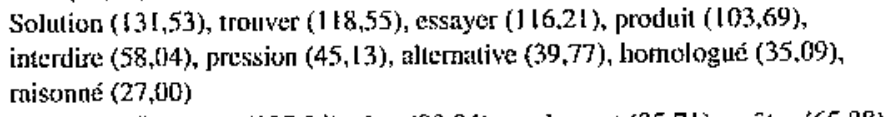 \\
\hline 4. Rendement el pesticides & $\begin{array}{l}\text { Bio }(208,25) \text {, penser }(127,24) \text {, clier }(99,84) \text {, rendentent }(95,71) \text {, conter }(65,88) \text {, } \\
\text { agricultufe }(59,00) \text {, avenif }(52,48) \text {, conventionncl }(48,48) \text {, prix }(37,30)\end{array}$ \\
\hline $\begin{array}{l}\text { 5. Déshethage, pesticides et } \\
\text { méthodes d' agriculture }\end{array}$ & $\begin{array}{l}\text { Herthe }(287,90) \text {, champ }(160,43) \text {, pousser }(134,08) \text {, lere }(125,05) \text {, récollc } \\
(90,19) \text {, matuvis }(77,85) \text {, caltiver }(63,91) \text {, beau }(60,94) \text {, haister }(50,57), \\
\text { désherbage }(46.10)\end{array}$ \\
\hline
\end{tabular}

thématique « associations de mots $»\left(\mathrm{Chi}^{2}=306,65\right)$, dans la mesurc où les interviewós ont été invités, en début d'entretien, à expliciter des associations de mots produites autour du mot inducteur « pesticides $*$ lors de la passation d'un questionnaire préalable. Ainsi, dans cette classe 1 , on trouve les éléments jivrés de tmanière spontanée et structurant leur représentation des pesticides. Il y est guestion de la pollution (Chi $\left.{ }^{2}=192,89\right)$, des effels des pesticides $\left(\mathrm{Ch}^{2}=143,44\right)$ qui sont plutôt néfastes $\left(\mathrm{Chi}^{2}=67,74\right)$. La nature $\left(\mathrm{Chi}^{2}=40,41\right)$ et l'environnement $\left(\mathrm{Chi}^{2}=49,79\right)$ sont atlaqués $\left(\mathrm{Chi}^{2}=35,55\right)$. Les pesticides sont quatifies de poison $\left(\mathrm{Clai}^{2}=60,05\right)$; ils sont associés at fait de tuer $\left(\mathrm{Chi}^{2}=68,67\right)$, à la testruction du vivant (respectivement $\left.\mathrm{Chi}^{2}=61,36, \mathrm{Chi}^{2}=60,05\right)$. Notons toutefois qu'un discours sur la destruction de la faune ef de la flore est akssi associé pour certains segments de texte à une destruction des « ravageurs », dans le but de protéger les cultures.

Les pesticides, englobant dans les discours les herbicides, les fongicides et les insecticides, sont présentés comme des produits puissants, loxiques, pouvant détruire toute la vie du sol, de manière plus ou moins profonde, touchan la litière, la première couche du sol, ou se propageant davantage. Ceux-ci sont présentés comme étant dangereux pour la nature. Un interviewécompare à ce propos le rapport entre les pesticides et l'environnement à un combat disproportionné : « les pesticides sont les ennemis de l'environnement, c'est David et Goliath * ( $\mathbf{n}^{\circ} 21$, étudiant, 18 ans, Martinique).

La position quant à la toxicifé des pesticides dans les discours varie selon la perception de leur (in) capacité à cibler ( $\left.\mathrm{Chi}^{2}=45,74\right)$, ce qui doit être détruit de ce qui doit être préservé. Certains mentionnent les produits sanitaires conme ćtant capables de a cibler certaines plantes, certaines espèces " ( $\mathrm{n}^{\circ} 50$ étudiant, 18 ans, Bretagne) ou bien argumentent que la propagation est fonction du savoir-faire, de la manière d'appliquer le produit. $A$ l'inverse, l'effet néfaste des pesticides est relié à tar représentation des pesticides comme quelque chose de non-sélectif, qui détruit tout de manière massive dans le sol, sans distinction, qui « déséquilibre le milieu » (agriculteur, 57 ans, Martinique, Agriculture bio). Cette destruction concerne la faune $\left(\mathrm{Chi}^{2}=97,69\right)$, qui se réduit cependant aux insectes et la fiore $\left(\mathrm{Chi}^{2}=114,64\right)$. La forme abeille $\left(\mathrm{Chi}^{2}=55,9\right)$ est particulièrement citée. Les interviewés mentionnent l'mmact sur le sol $\left(\mathrm{Chi}^{2}=62,95\right)$, sur la qualité de l'eau des rivières $\left(\mathrm{Chi}^{2}=17,3\right)$ ou des nappes phréatiques $\left(\mathrm{Chi}^{2}=47,13\right)$. Par exemple, le danger de contaminer les cours d'eau en cas de forte pluie est mentionné. 
Le monde lexical de cette classe correspond spécitiguement à un discours sur la propagation de la pollution, d'une réaction en chaine. I1 ne s' agit plus d'un ensemble d'effets mais d'une relation entre ces différents effets : $\alpha$ c'est comme lorsque vous jetez une pierre dans j'eau avec les ronds $*$ ( $\mathrm{r}^{\circ} 38$, agriculteur; 45 ans, Bretagne, agriculture raisonnce). C'est l'écosystème qui est touché par les pesticides, écosystème au sein duquel l'homme trouve place même si les liens entre la santé de l'bonme (respectivement $\mathrm{Chi}^{2}=42,54, \mathrm{Chi}^{2}=132,95$ ) et la qualité de l'environnement sont timides. De plus, l'intégration des êtres humains dans cet écosystème, impliquant de fait des conséquences pour la santé, est évoquée principalement (et de manière quasi-exclusive) par les interviewés résidant en Martinique, avec plus ou moins de nuances:

«Si le produit tue un insecte, il peut nous tuer aussi. Tout va dépendre de la dose et de la fréçuence " $\left(\mathrm{n}^{\circ} 14\right.$, agricultrice, 43 ans, Martinique, agriculture conventionnelle).

«On tue tout ce qui est élément vivant dans le sol, donc l'environnement est attaqué et la santé le sera par la suite. [...] Tout le microsystème est là. [ . . . Le fait d'avoir utilisé des pesticides dans les bananeraies, notamment le chlordécone, a donné un sol empoisonné pour plus de 150 ans ainsi que les problèmes de cancer de la prostate, de stérilisation des hommes, des femmes qui n'enfantent pas, etc. On n'esi pas près d'en finir $"\left(\mathrm{n}^{\mathrm{0}} 2\right.$, agriculteur, 50 ans, Martiniçue, agriculture bio).

En Martinique, la pollution des sols est en effet principalement liée à l'utilisation du Chlordécone ${ }^{2}$; les interviewés y font référence à titre d'exemple, voire d'avertissement pour j'avenir.

\subsubsection{Les risques liés à l'utilisation des pesticides pour les humains}

La classe 2 est centree sur les risques encourus (ou non) par les hommes. Le dikcours des étudiants est significativement présent dans cette classe ( $\mathrm{Cbi}^{2}=41,48$ ). Il y est surtout question des agriculteurs qui doivent se protéger, notanment en utilisant les équipements de protection recommandés. A contrario, les interviewés ne soni pas très prolixes, pour ne pas dire complètement silencieux, sur les risques encourus par les consommateurs. Aucane mention n'est faite des résjdus de pesticides dans les produits agroalimentaites ou alimentaires - dont l'ingestion est néfaste pour la santé - bien que ce sujet soit très présent dâns les médias.

Les « utilissateurs » sont présentés avant tout comme étant responsables de leur propre santé. Ce n'est pas tant la dangerosité des produits phytosatitaires qui est mise en cause, mais un manque de précaution de la part des « humains »: «C'est prouvé scientifiquement que, d' une part, les gens qui trilisent ces produits-là ne sont pas bien protégés et peuvent être très malades 》 ( $\mathrm{n}^{\circ} 42$, étudiante, 20 ans. Bretagne $)$. Les formes protéger $\left(\mathrm{Chi}^{2}=121,07\right)$ et protections $\left(\mathrm{Chi}^{2}=135,06\right)$ sont significativement présentes. Le discours est lié à l'utilisation $\left(\mathrm{Clhi}^{2}=2=181,38\right)$ et au traitement $\left(\mathrm{Chi}^{2}=50,94\right)$. Ce qui est remarquable, c'est l'association dans une même classe đes discours sur les risques liés à l'utilisation des pesticides et sur la connaissance de la réglementation de

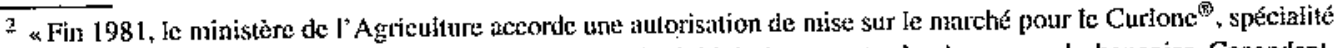
contennal sgalenent $5 \%$ de chlordécone. Son tsage tust limifé à la lutie contre le charançon du bananicr. Cependant, la persistance des organochlores dans l'enwironnenelti et leur foxicité pour certains brgarismes vivants arvail conduil

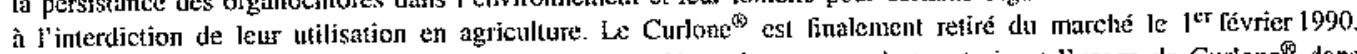
Néanmoins, après l'artêt d’auterisation de vente deux dérogations succossives autorisent l'usage de Curtone dans les DOM jusqu"en septembre 1993. Au lotal, près de 300 tonthes de chlordecone (soil 6000 tontes de Curlone ont diłsj cté ulilisces aux Antilles entre 1981 et $1993 \%$. Site de l'Observatoire des résidus de pesticiues (ORPS),

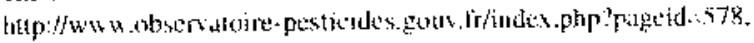


l'usage des pesticides, soulignée à travers l'utilisation des formes dose $\left(\mathrm{Cli}^{2}=53,99\right)$, équipement $\left(\mathrm{Chi}^{2}=38,51\right)$ et protection $\left(\mathrm{Chi}^{2}=135,06\right)$. Les deux variables thématiques, respectivement «risques liés à l'utilisation de pesticides», $C h^{2}=80,02$ ) et «réglementation de l'utilisation des pesticides $»\left(\mathrm{Chi}^{2}=57,43\right)$ sont présentes significativement dans cette classe. Le tisque semble être linité ì la dose de produit utilisée et à son contact lors de l'utilisation: "c'est comme quelqu'un qui va furner trois cigarettes par jour ou un paquet par jour " ( $\mathrm{n}^{\circ} 59$, agriculteur, 40 ans, Sud de France, agriculture conventionnelle), $\propto$ j] faut utiliser la "bonne dose au bon moment" ( ${ }^{\circ}$ 34, ag;iculteur, 56 ans, Bretagne, agriculture raisonnée). Les interviewés rappelleni tes reconmandations indiquées sur les étiqueties des produits.

Ainsi, dans la classe 2, ce n'est pas la toxicité des pesticides qui est incriminée, mais le nanque de précaution lors de Ieur utilisation. Les risques paraissent relatifs. Bien entendu, ce point de vue n'est pas partagé par tous. Certains interviewés (notamment ceux en production bio) font part de teur étonnement, partagent leur irritation quant au fast d'utitiser un produit qui est mentionné comme étant dangereux, surtout quand celuj-ci arbore une «tête de mort ». D'autres, spécifiquement les interviewés de Martinique, soulignent le manque de clarté dans lá réglementation ou tien son non-respect par les utilisateurs. Ils forit rélérence ici aussi à la pollution engendrée par le Chlordécone ( $\mathrm{Chi}^{2}=29,57$ ) qu'tls associent au cancer $\left(\mathrm{Chi}^{7}=32,42\right)$. Les risques sont aussi présentés comme étant méconnus. Un manque d'information peut être cité comme cause. Pour certains étudiants, c'est un manq̨ue dans leur formation ou au niveau des médias qui n'en parlent pas assez. Le manque $d^{*}$ information peut aussi être vu conme un « tabou » $\left(\mathrm{n}^{\circ} 28\right.$, étudiant, 22 ans, Martinique), on comme stant délibéré afin de ne pas faire peur aux poputations.

\subsection{Pratiques et pesticides}

Les classes 3,4 et 5 concernent les pratiques on hien avec les pesticiodes. Il s'agit des pratiques actuelles mais aussi des pratiques futures, des alternatives possibles, celles qui sont envisageables et/ou envisagées. Les interviewés s"expriment notanment sur l'avenir des pesticides dans la classe 4 «rendement et pesticides》 et dans la classe 3 «des alternatives aux pesticides ». La variable thématique « question sur l'avenir des pesticides » est présente significativement dans ces deux dernières classes (classe $3: \mathrm{Chi}^{2}=56,27$; classe $4: \mathrm{Chi}^{2}=40,15$ ).

\subsubsection{Les mawaises herbes, ennemies ou alliéer des agriculteurs?}

La classe 5 englobe des mondes lexicaux inhérents au travail de la terre, au type de culture réalisée, en lien avec l'utilisation ou non de pesticides. Il s'agit avant tout du discours des agriculteurs $\left(\mathrm{Chi}^{2}=89,83\right)$. La variable thématique Métier associée aux segments de texté décrivant l'áctivité des agriculteurs, est présente significativennent $\left(\mathrm{Chi}^{2}=65,29\right)$. La description du métier exercé est en lienl avec le monde lexical de cette classe 5 . Les personnes procédant à un type agriculture conventionnelle $\left(\mathrm{Chi}^{2}=38.54\right)$ et à un type agricalture bio $\left(\mathrm{Chi}^{2}=31,93\right)$ sont présentes dats cette classe au contraire de ceux privilégiant une agriculture raisonnée.

On trouve, dans la constitution de cette classe, des formes lexicales propres an type de producfion zćalisée, telle que la forme maïs $\left(\mathrm{Ch}^{2}{ }^{2}=158,84\right)$. La part la plus importante de cette classe, ainsi que sa spécificité, résident dans un discours sur la position envers les mauvaises herbes (respectivement $\mathrm{Chi}^{2}=77,85, \mathrm{Chi}^{2}=287,9$ ), et sur le fait qu'il faille ou non les laisser pousser (respectivement $\mathrm{Chi}^{2}=50,57, \mathrm{Chi}^{2}=134,08$ ). Elles sont présentées comme étant néfastes aux cultures, à la qualité des champs $\left(\mathrm{Chi}^{2}=160,43\right)$ ou des récoltes $\left(\mathrm{Clii}^{2}=90,19\right)$ - dans ce cas fortement corrétées à l'image, à l'apparence, à ce qui cst beau $\left(\mathrm{Chi}^{2}=60,94\right)$ comme « la belle 
pomme de ta Belle au bois domant $\gg\left(\mathrm{n}^{\mathrm{0}} 30\right.$, agriculteur, 45 ans, Bretagne, agriculture biologique $)$. Les pesticides ont ici une fonction importante - la forme herbicides $\left(\mathrm{Ch}^{2}=4,08\right)$ est présente dans cette classe - ils permettent de lutter contre l'envahissement des cultures. Des techniques alternalives aux pesticides, utilisées on envisageables, sont aussi mentionnées, telles que laisser les feuilles coupées pour empêcher que l'herbe ne pousse. De plus, le fait de ne pas désherber chimiquetrent présente des avantages sur les cultures. Par exemple, des agriculteurs racontent que leurs champs avec des mauvaises herbes se font moins attaquer par les insectes, que le sol est plus riche et les récoltes meilleures. Toutefois, ceux qui font le choix de laisser des herbes dans leurs champs partagent leur sentiment d'être rẹjếs,s, stigmatisés. Une agricultrice bio revient sur cette image négative : « J'ai un champ qui est bio. Il y a des mauvaises herbes et les gens du villagc le savent et ils disent, jis parlent de mon champ $\gg\left(\mathrm{n}^{\circ} 60\right.$, agricultrice, 38 ans, sud de la France, agriculture biologique). D'autres, notamment ceux privilégiant une agriculture raisonnée, argumentent en faveur d'une utilisation minime de pesticides plutôt que leur interdiction totale.

\subsubsection{Peut-on se passer de pesticides à l'avenir? Une question en lien avec les rendements} nécessaires

La classe 4 est la plus importante en termes de segments de textes classés : $20,6 \%$. L'avenir des pesticides est le thème central de cette classe (avec un $\mathrm{Ch}^{2}=56,27$ pour cette variable thématique). Les interviewés partagent leurs avis (le verbe penser a un $\mathrm{Chi}^{2}$ élevé : $\mathrm{Chi}^{2}=127,24$ ).

Le monde lexical de la classe 4 répond en quelque sorte à l'interrogation suivante: est-i) possible $\left\langle\mathrm{Chi}^{2}=35,13\right)$ de se passer $\left(\mathrm{Chi}^{2}=[16,12)\right.$ de pesticides à l'avenir $\left(\mathrm{Chi}^{2}=52,48\right)$ ? Cente réflexion engendre un discoutrs sur $l^{2}$ agriculture bio $\left(\mathrm{Ci}_{1} i^{2}=208,25\right)$ et ses différences avec l'agriculiture conventionnelle $\left(\mathrm{Chi}^{2}=48,48\right)$. Dans la plupart des cas, les interviewés énoncent ce mode d'agriculture comme étant cité à tort comme une solution. L'argumentation vise à soulfgner ses failles. Le fait de pouvoir se passer de pesticídes est mentionné comme dépendait de variables contextuelles, à savoir le lerritoire et la taille de l'exploitation. Toutefois, le discours se cristallise spécifiquement autour d'un point précis : la question des rendements $\left(\mathrm{Chi}^{2}=95,71\right)$. Les pesticides y jouent un rôle en assurant une productivité suffisante.

Les rendements sont associés à deux discours. Premièrement, un discours qui porte sur la rentabilité đe l'exploitation, c'est-à-dire en termes de coûts et bénéfices. La thématique économiqué est saillante dans cette classe. On observe tes formes lexicales suivantes : coûter $\left(\mathrm{Chi}^{2}=65,88\right)$, prix $\left(\mathrm{Chi}^{2}=37,3\right)$, argent $\left(\mathrm{Chi}^{2}=33,96\right)$, gagner $\left(\mathrm{Chi}^{2}=32,93\right)$, payer $\left(\mathrm{Chi}^{2}=16,41\right)$. Une agriculture biologique. donc sans pesticides, est présentée comme êtant plus coûteuse, moins rentable. D'ailleurs, des tenants de ce discours pointent le fait que ceux qui privilégient l'agriculture bio ne peuvent vivre que par les subventions ou les aides. Deuxièmement, la question des rendements peut surpasser la dimension économique, individuefle, et être mobilisée pour souligner une inadéquation de l'agriculture biologique avec la fonction première du métier, à savoir, nourrir tous les hommes. Il s'agit de l'avenir de la planète $\left(\mathrm{Chi}^{2}=29,1\right)$. Les rendements assurés par une production bio ne sont pas perçus comme étant suffisants pour nourrir $\left(\mathrm{Chi}^{2}=7,37\right)$ tout le monde:

«Pour nourrir la planète, ça va être difficile sans les pesticides » ( $n^{0} 56$, étudiant, 21 ans, Bretagne).

« Honnêtennent je pense que si demain tous les agriculteurs du monde se mettent au bio, dans un an on suce des pierres " ( $n^{\circ} 63$, agriculteur, 45 ans, sud de la France, agriculture conventionnelle). 
Ce lien entre rendements faibles et agriculture biologique est remis en question par certains, qu'ils soient en agriculture bio ou pas. Cette position est présentée conme étant liée à un manque d'informations, voire ande rétention d'informations. Des freins au changement sont cités, notamment en lien avec des habitudes: ac'est vrai que le conventionnel nourrit la France mais ils devraient essayer l'autre système et ils verront, ça peut marcher $\gg\left(n^{\circ} 58\right.$, agriculteur, sud de la France, agriculture bio), Pour argumenter, des exemples d'agriculteurs ayant opté pour la culture bio et étant tout à fait satisfaits, voire des exploitants ayant augntenté leut's bénéfices, sont cités.

\subsubsection{Des altenatives aux pesicicies}

La classe 3 concerne également l"avenir des pesticides. Elle est liée à un discours sur la perception des agriculteurs par rapport à leurs propres pratiques vis-à-vis des pesticides. La variable thématique «utilisation des pesticides » présente un $\mathrm{Chi}^{2}$ de 30,3. L'utilisation de pesticides est expliquée seton un ensemble de critères visant à assurer de bonnes pratiques. Tour d'abord, un discours est produit sur la réglementation, les clroits $\left(\mathrm{Chi}^{2}=28,85\right)$, les recommandations à suivre, les produits autorisés ( $\left.\mathrm{Chi}^{2}=29,34\right)$, homologués $\left(\mathrm{Chj}^{2}=15.73\right)$. Les pratiques sont présentées comme étant encadrées $\left(\mathrm{Chi}^{2}=19,94\right)$. Les agriculteurs soulignent le fait qu'ils suivent une dématche qui leur est conseillée par des professionnels de l'agriculture, que ce soit pout les produits à utiliser ou les principes de précaution à suivre. La variable thérnatique a conseils sur les pesticides » est également présente significatjvement daus la classe $3\left(\mathrm{Chi}^{2}=16,39\right)$. L' utilisation est représentêe conme ćtant scrupuleusement régie par des règles précises et strictes. Unie autre différence est soulignée : l'utilisation mussive de pesticides a globalement fortement diminué el devlaait 1'être plus encore à l'averir : « on n' utilise plus comme dans les annếes 1950, où on traitai de partout, c'est plus du tout pareil. Il y a des nomes bien strictes [.. .] Matitenant, c'est bien réglementé aussi » ( ${ }^{\circ} 55$, étudiant, 22 ans, Bretagine).

Des intervjewés énoncent leur élan vers la réduction de passitges de produits phytosanitaires sur leurs récoltes. La méthode alternative n'est pus de supprimer les pesticides mais de limiter leur utilisation, notamment par une diminution des doses.

Si l'avenir des pesticides est lié, dans cette classe, à des doses limitées (Chi $\left.{ }^{2}=22,04\right)$, cette classe répond majoritairement à la question suivante: « quelles attres solutions que les pestjcides?\%. Il est question des alternatives $\left(\mathrm{Chi}^{2}=39,77\right)$, des solutions $\left(\mathrm{Chi}^{2}=131,53\right)$ face à une Eventuelle interdiction (Chi $\left.{ }^{2}=10,19\right)$ des pesticides. Notss pouvons observer que les discours concernent ce qui est ou devrait être envisageable dans le futur: « on essaye de trouver des solutions » (agriculteur, 46 ans, sud de la France, agriculture conventionnelle). Le verbe essayer présente un $\mathrm{Chi}^{2}$ des plus élevés de la classe $3\left(\mathrm{Chi}^{2}=116,21\right)$. Les solutions ne sont pas encote la, il fatt les trouver $\left(\mathrm{Chi}^{2}=118,55\right)$, notamment des solutions qui permettrajent de concilier le respect de l'environnement et les besoins et attentes des agriculteurs : « il faut laisser la place à la science pour faire le clair, mais it ne fatt pas laisser les paysans sur le bord de la route $\$\left(\mathrm{n}^{0} 38\right.$, agriculteur, 45 ans, Bretagne, agriculture raisonnée, $\mathrm{Clii}^{2}=271,24$ ).

De plus, l'utilisation oil non de pesticides, les quantités et les produits autorisés, loutes ces normes sont associées, spécifiquement chez les agricultears bio, à un discours. sur le business $\left(\mathrm{Chi}^{2}=2,09\right)$, fes lobbies (Chi $\left.{ }^{2}=20,4\right)$ qui dictent les temdances, qui crêent une demande, voire une dépendance :

«Je pense que les paysans sont bloqués par les Jobbies, parce qu'à partir du moment où on a créé un produit qui va tuer toul un écosystème, du coup ga va faire découler plein de problènes. Enfin, on a eu une attaque de pucerons, on a tué les pucerons mais on a tué tout le monde, donc on va se retrotiver avec la chenijle qui va arriver derrière et du coup, on revend 
des produits toujours pour contrecarrer un problème alors que te puceron n'était peut-être pas un problème à la base » ( $n^{0} 30$, agricultew, 45 ans, Bretagne, agriculture biologique).

De plus, un manque d'équité est énoncé lorsque les normes quant à l'utilisalion des pesticides sont pensées à une pỉus large échelle. Par exemple, lorsque des produits sont interdits en France, ils sont vendus ailleurs.

Dans les alternatives, si l'agriculture ou les produits bios sont cités, ils ne sont pas systématiquenent présentés comme étant une solution adaptée. Certains interrogent l'efficacité des «solutions naturelles $*$, d'autres mentionnent le fait que les produits biologiques polluent autant que les autres. La pulvérisation à base de cuivre est principalement citée que ce soit chez les agriculteurs ou chez les étudiants : « Tout ce qui est cujvre et tout, enfin, ça pollue beaucoup touł ce qui est sol et tout. [Il faudrait] te limiter, parce que ça aussi ça va plus polluer qu' un produit de synthèse qu'on va utiliser » $\left(\mathrm{n}^{\circ} 74\right.$, étudiant, 20 ans, sud de la France).

Ainsi, si certains interviewés évoquent le besoin de trouver des ahernatives pour les remplacer $\left(\mathrm{Chi}^{2}=34,97\right)$ on pour s'en passer en amenant l'écosystème à se réguler tout seul satts l'intervention de produits, peu de solutions concrètes sont cilées. Ils mentionnent la préférence pour les insectifuges plutôt que les insecticides, l'introduction de prédateurs naturels ou encore la conservation de la biodiversité, sans préciser pour autant les moyens d'y arriver.

\section{Discussion et conctusion}

Un des objectifs de cette étude étail de comparer ies discours en fonction du type de pratiques des personnes interrogées, que ce soit en termes d'expérience professionnelle (étudiants vs. agriculteurs en exercice) ou en termes d'implication retative à l'objet pesticides, Faisant ici référence au type d'agriculture (conventionnelle vs. ráisonnée vs. biologique). S' intéresser aux liens existant entre pratiques et représentations n'est pas nouveau. De nombreux travaux fon état du mouvement pendulaire entre, d'une part, les représentalions sociales et, d'autre part, les pratiques sociales que les groupes adoptent vis-à-vis d'un objet de représentation (Abric. 1904 : Jodele1, 1989: Moscovici. 1961). Dès lors, J'objectif affiché dans cette étude est d'intertoger ce lien entre les diffërentes pratiques liées à l'usage des pesticides et les représentations sociales qui leurs sont associées.

C'est ainsi que dans ce contexte, on peut relever que les différences majeures quant aux représentations sociales des pesticides selon les groupes interrogés, et a fortiori leur pratiques, concernent principalement le risque lié à ces produits.

Les résultats montrent que les étudiants évoquent davantage la question des risques encourus que les agricuteurs. Ces derniers soulignent surtout les moyens de protection et les principes de précaution à appliquer. Des distinetions sont cependant observables au sèin du groupe des agriculteurs.

Les agriculteurs favorisant une mode de production biologique mentionnent significativement ces précautions comne étant insufinsantes et soulignent la dangerosité des produits phytosanitaires pour l'utilisateur et le consommateur. Les agriculteurs en Martinique présentent un discours spécifquement lié à cette variable contextuelle.

Poư argumenter la toxicité des pesticides, ils se réfêrent à l'expérience de poltution des sols en Martinique par le chlordécone tout en ajoutant que la réglementation ne peut suffire à elleseule. Ce constat est cohérent avec les travaux initiés par Ruuqusette (1997) sur les différents types d'implication qui influencent les représentations sociales. Eu égard, le contexte spécifique lié au scandale sanitaite de la chlordécone, les agriculteurs martiniquais se situent dans une implication 
circonstancielle qui renvoie à un contexte spécifique en lien avec l'objet (i.e. être agriculteur en Martinique implique de fait les sujets vis-à-vis des risques sanitaires liée à l'utilisation des pesticides). De plus, si les propos sur l'impact environnemental de l'utilisation de pesticides ef sur les risques encourus lors de leur utilisntion sont principatement tenus par les étudiants, cela tae signifie pas qu'ils pointent plus précisément un effet néfaste des pesticides que les agriculteurs mais qu'ils mentionnent plus significativement les interrogations que le sujet suscite. À ce propos, on peut noter que la formation semble être un facteur ayant peu de poids clans. la fornulation des ides. Les étudiants ne mentionnent pas d'éléments acquis lors de leur cursus. S'ils énoncent que les informations quant à ce sujet sont absentes de leur formation, elles sont en même temps très peu attendues ou réclamées. Toulefois, certains d'entre eux témoignent de l'ćvolution de leur opinion, notamment relative aux cultures biologiques lorsqqu'ils ont été en contact avec des agriculteurs bio lors de stages.

Les entretiens, indistinctement ceux des agriculteurs et des étudiants, sont ponctués d'exemples, de cas particuliers. Les interviewés se réferent surtout à ce qu'ils ont entendu dans les médias, lors de conversations quotidiennes ou par le partage d'expériences directes ou indirectes. Antcune information ou plus générale n'est utilisée pour argumenter, que ce soit d'ujleurs chez ceux qui privilégjent "'agriculture bio ou chez ceux qui mettent en avant son manque d'efficacité.

Précisément, le discours des interviewés quant aux risques liés à l'utilisation des pesticides, toutes variables confondues, est propre à la logique naturelle (Rouquette. 2009), c'est-à-dire qu'ils font appel à des théories expertes mais anonymes, à des observations, à leurs intuitions, à des đéductions ou encore à des expériences transmises ou vếcues. Ils font, par exemple, référence aux documentaires ou témoignages diffusés dans les médias.

Ainsi, les discours sur les pesticides ne renvoient pas à un savoir expert. Il est icí importan de mettre en garde contre une conception biérarchique d'un savoir expert versus profane liéc à la connaissance d'utilisation des pesticides. En effet, le savoir profane n'est pas un non-savoir, mais « un système de représentations organisé et structuré selon des lois qui lui sont propres» (Caillaud \& Kalampalikis. 2(1)(0).

Les interviewés ne semblent pas intégrer l'usage, et sturtouk le bon usage des pesticides, conme une compétence spécifique de leur méticr. Ils parlent des pesticides comme d'un objet aux contours fous. Le discours autour de la propagation des pesticides en est une illustration marquante. Celleci est un plénomène assez flou et parcellaire dins la majorité des discours. Si le fait que les pesticides puissent circuler de parcelle en parcette via l'eau lors de fortes plities, leur propagation dans le sol et dans les récoltes est peu explicite et est avant tout présentée comme étant peu diffuse. Nous avons recueilli des résultats similaires dans une autre recherche ${ }^{3}$ portant sur les représentations et pratiques en lien avec les produits phytosanitaires, mais dans ce cas chez des jardiniers anłateurs. La pollution des sols ou de l'eau par les pesticides est représentée comme quelque chose de contenu, de limité dans l'espace. L'écosystème n'est pas pensé dans sa globalité et dans sa temporalité (Levahseur. Cerisier. \& Hass, 2013). D'autres auteurs soulignent que la profession agricole a un faible niveau de perception de l'impact réel dé ses activités sur la qualité de l'eau (Rousirary, Buscu. Salles. Dumont, \& Carpy-Gesilard, 2013).

Les résultats tendent à montrer quẻ les interviewés ne se sentent pas investis dans la commıınjcation autour des risques. Pour les agriculteurs ou ceux qui se destinent à ce métier, les pesticides ne font pas partie de leur domaine de compétences en leur discours vise à appuyer et étayer cette

\footnotetext{
7 * Des cinercheturs au jardir, quand la science se utel au serrice de fa demande sociale $\%$, GRePS en collaboration awe l'équipe du service science et société du PRES de Lyon, IRSTEA er la FRAPNA (financements; région Rhône-Alp̣es, appel ì projel a université citoyeline et solidaire) (2012-2013). Synthèse du rappon de recherche en ligne.
} 
prise de distance. If́s mentionnent les vendeurs qui leur donnent des conseils sur les produits sanitaires comme étant les experts du domaine, ainsi qu* une réglementation à suivre. Les agriculteurs justifient $l^{\prime}$ utilisation de pesticides par les autorisations existantes. Les règles à suivre sont énoncées. Dès lors, si l'existence de risques est évoquée, ces demiers peuvent ĉtre circonstanciés. En somme, si les utilisateurs utilisent les équipements de protection individuelfe el respectent les doses, les risques sont misimes, voire inexistants. L'évaluation du tisque se fait aussi en comparáson avec les décennies précédentes pour souligner les progrès en termes de toxicité des prodnits. Les progrès réalisés sont davantage cités que les précautions actuelles ou la nécessité de réduire l'utilisation de produits phytosanitaires. Roussary at at. (2013) ont obtenu des résultats similaires. Ifs ont également montré que lat prescription affichée par le fabricant prend valeur de « bonne pratique » (p. 76). Ainsi, la revendication d'une responsabilité partagée, voire d'une externalisation de responsabilité est régulièrement mobilisée par les agriculteurs (Roussary et al.. 201 3, p. 74).

\section{Terre vivante, terre cultivée : une opposition cristallisant les positions quant à la nécessité des pesticides}

Les discours sur les pesticides présentent une opposition enire la ierre "vivante » et la terre agricole, cultivée. Cette conception renvoie, d'une part, à une terre maitrisée par l'honme, celle de ja culure, qui peut dans les discours de ceux présentant les pesticides comme étant toxiques pour les sols, n'avoir plus rien de vivant, et de l'autre à une terre «naturelle \$ dans le scas de non-transformée, mais qui va être moins contrôlable et contrôlée. Cette dualité semble assoir les prises de position quant à l'utilisation des pesticides. Le discours tenu sur les pratiques face aux mauviuses herbes cn est révélateur. Une parcelle «propre», c'est-à-dire ne présentant pas de mauvaises herbes, tenvoie à une terre cultivée tandis que, comme l'ont montré récelnment Roussary el al, (2013), «la présence d'adventices ou de nuisibles - sur ses propres cultures ou dans les parcelles voisines - renvoie pour les agriculteurs à une stigmalisation de a mauvaises pratiques agricoles * el du « laisser-aller . Cette représentation de la terre cultivée comme devant être orỏonnéc, rattachée à une identité sociale positive pour la majorité des agriculteurs s*exprime dans les pratiques. Désherber ses terres est une pratique signifiante.

Le fait que la pratique de techniques alternatives à l'utilisation des pesticides, assimilée la plupart du temps à une culture bio ou au fait de ne pas recourir à un dẹ́sherbage systématiçue. soit stigmatisée est mentionnée par les interviewés. De plus, te fait de ne pas utiliser de pesticides est surtout associé à un surplus de travail. Des agriculteurs mentionnent ce travail supplémentaire comme étant à l'encontre d'un des objectifs principaux : optimiser le rendement. Ainsi, le discours autour clu désherbage est un bon révélateur du positionnement du sujet par rapport à l'ituilisation et l'utilité des pesticides.

Ainsi, les résultats montrent que la thématique des enjeux environnementaux est bien distincte de celle propre aux pratiques professionnelles. De plus, les perspectives, englobant la nécessité de faire évoluer les pratiques et les solutions envisageables, quj sont bien souvent assimilées et limitées à l'agriculture biologique, sont de l'ordre d'un discours avancé comme érant une question pour les générations fulures. Toutefois, même si les étudiants sont plus enclins à pointer cette problématique que les agriculteurs - à l'exception de cenx en production biologique- la diminution de l'utilisation des. pesticides, par l'emploi de méthodes alternatives d'agriculture, n'apparaît pas comme une question centrale aujourd'bui. Ce sont avant tout les doses qui pourraient être (encore) réduites. Les produits phytosanitaires sont principalement représentés contume étant à l'heure actuelle irremplaçables, que ce soit pour ussurer des rendements suffisants, pour nourrir la planète, ou encore d'un point de vue économique pour les agriculteurs. 
Dès lors, si la question des pesticides peut être mentionnée comme un aspect problématique au sein de la société, leur avenír n'est pas présenté majoritairement comme un des enjeux prioritaires de la profession. Plus précisément, la question des alternatives à l'usage des pesticides est représentée comme ótant l'apanage d'experts, dont les agriculteurs ne pensent pas faire partie, experts qui n'ont pas encore trouvé de solutions adéquates pour la société et satisfaisantes pour les professionnels actuels ou en devenir. Enfin, outre la théorie des représentations sociales, il convient également d'interroger ces résultats sous le prisme des représentations professionnelles. « Les représentations professionnelles sont des représentations sociales claborées dans l'action et la communication professionnelles (interagir et inter-réagir) et sont spécifiées par les contextes, les acteurs appartenant à des groupes et les objets pertinents et utiles pour ]'exercice des activités professionnelles » (Błin, 1997, p. 80). Dès lors, les représentations professionnelles font echo aux savoirs techniques que les individus, ici les agriculteurs, ont de leur métier et des outils associés. Les pesticides sont un objet utile s'inscrivant dans un cadre plus global lié à la profession d'agriculteur. Ainsi, il nous paraît pertinent, en vue de travaux futurs, d'explorer plus en profondeur les trois dimensions relatives aux représentations professionnelles tel que Blin (1907) les a définis. Les résultats de cette étude invitent à la réflexion concejnant les dimensions fonctionnelles, contextuelles et identitaires liées à l'utilisation des pesticides.

\section{Déclaration de liens d'intérêts}

Les auteurs déclarent ne pas avoir de liens d'intérêts.

\section{Remerciements}

«Action menće dans le cadre de l'APR 201 I du programme de recherche « évalıation el reduction des risques liés aux pesticides " piloté par le ministère chargé de l'Écologie, avec l’appuj financier de l'ONEMA, par les cródits issus de la redevance pour pollutions diffuses attribués au fuancement du plan Ecoplyto piloté par le ministère chargé de l'Agticilture».

\section{Références}

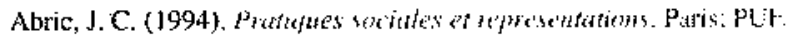

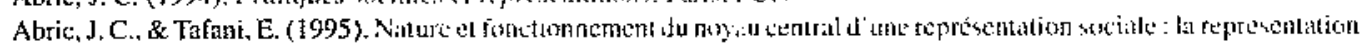

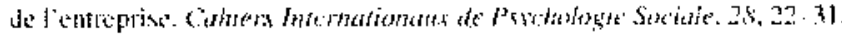

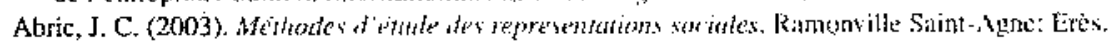

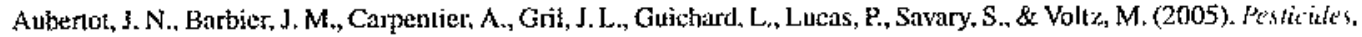

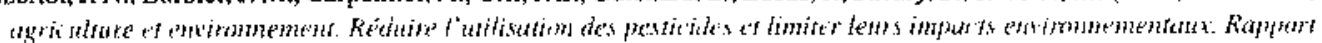

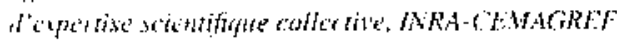

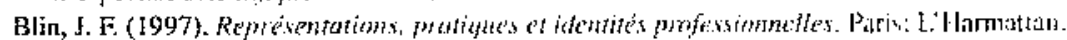

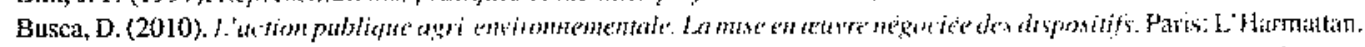

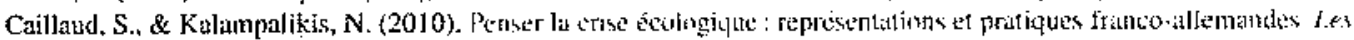

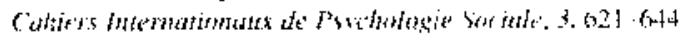

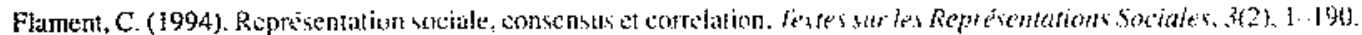

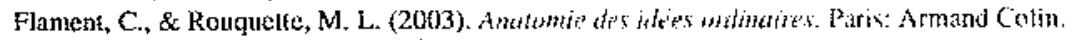

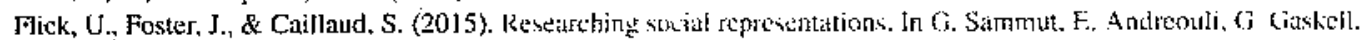

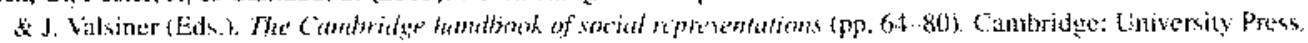

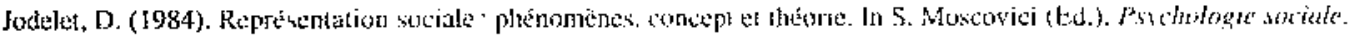
Paris: liti.

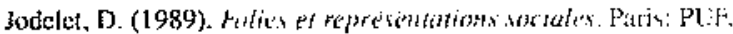




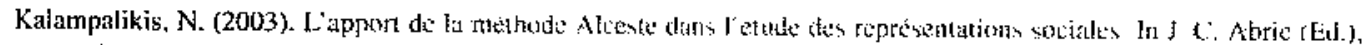

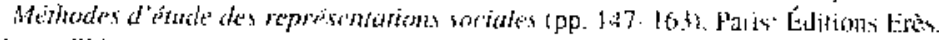

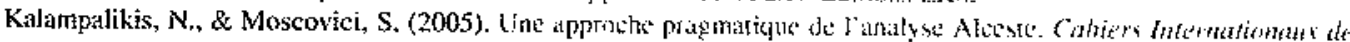
Martholmite Sucide.60, 1524

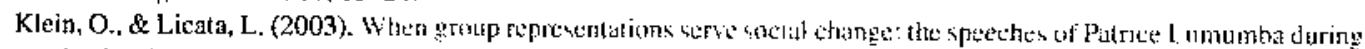

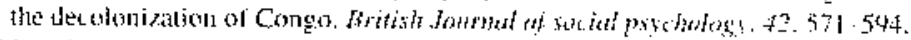

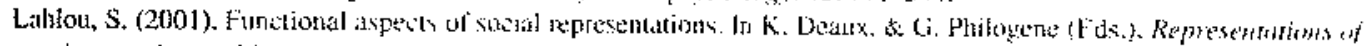

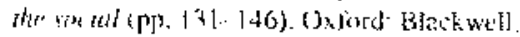

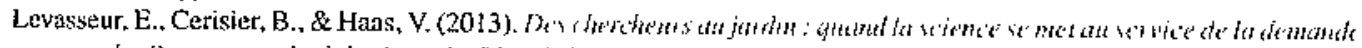

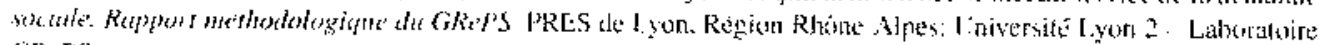
TReFS.

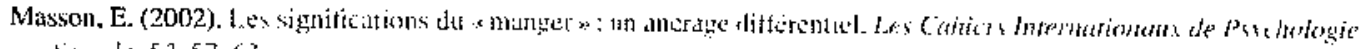
Sincruts. $59,57 \cdots 64$.

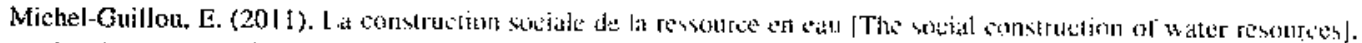

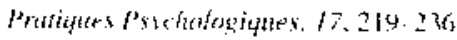

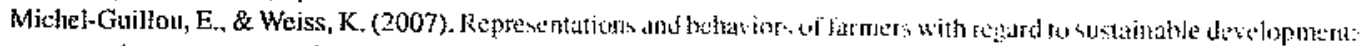

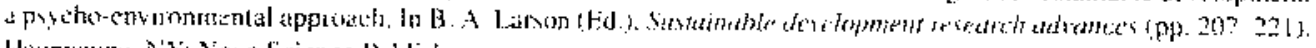

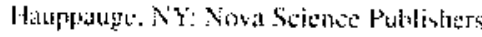

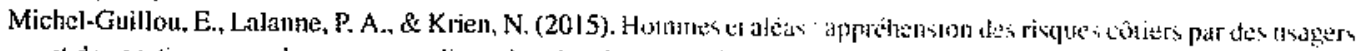

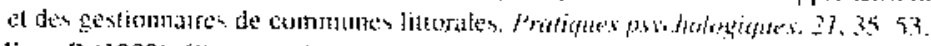

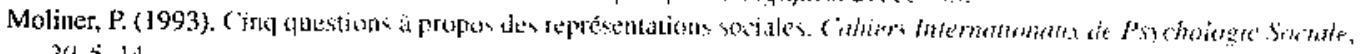
2). 3.14

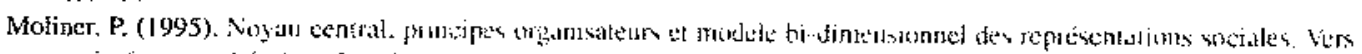

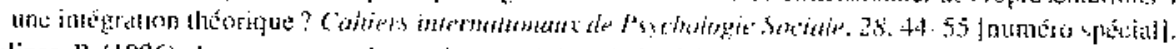

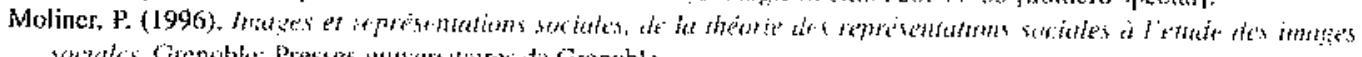

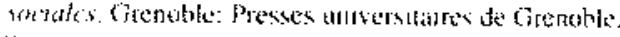

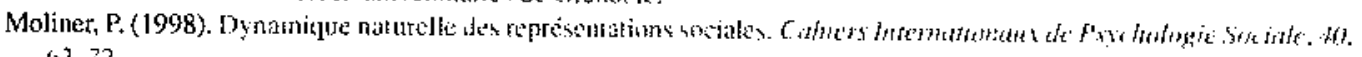
(1] ? ?2.

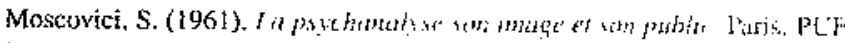

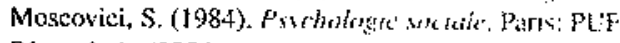

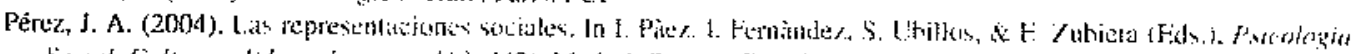

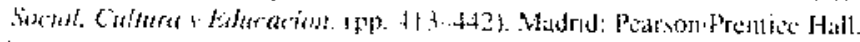

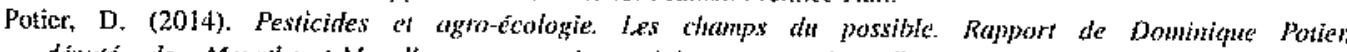

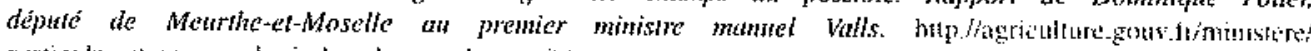

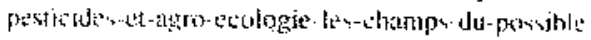

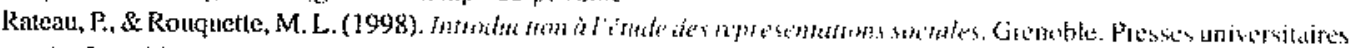
ito cirematis.

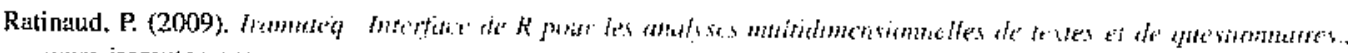
Whis; is

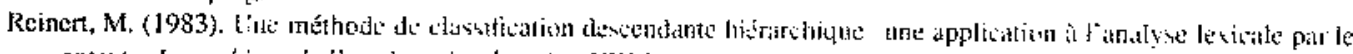

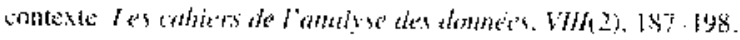

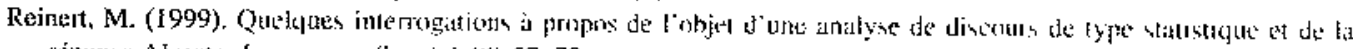

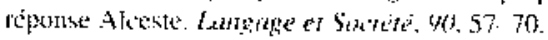

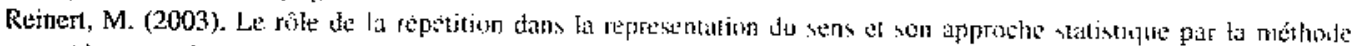

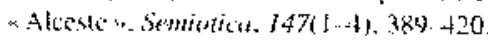

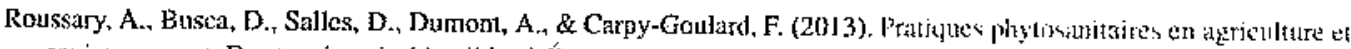

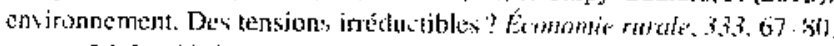

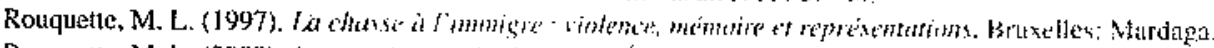

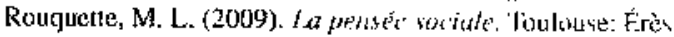

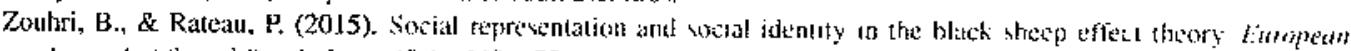

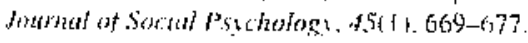

